\title{
CONDITIONS OF CRACKING OF A STRINGER
}

\author{
D. I. BARDZOKAS AND G. I. SFYRIS
}

Received 13 December 2002

In the limits of brittle failure of materials, we investigate the problem of possible cracking of an infinite stringer on the boundary of an elastic half-infinite plate. The plate is exposed to tensioning by uniformly distributed forces, and also to contact stresses due to application of forces on the stringer. The accurate solution of a contact problem of interaction of an infinitely continuous stringer is constructed. With the help of this solution, we formulate the condition of cracking of a stringer and the necessary restrictions for external loading, which provide the contacts of a broken stringer with a plate without propagation of a crack inside the stringer. The problem considered here is of interest for theory and practice. We firstly formulate the modified problem of E. Melan and with the help of Fourier integral transformation, we construct its acoustic solution and then formulate the condition of cracking of an infinite stringer. After that, reveal the necessary restrictions on external loadings, which provide the contact of the failed stringer with a plate without propagation of the crack inside the plate.

\section{Introduction}

One of the most important scientific and technical problems is the creation of structural materials with high rigidity, strength, and reliability. Such materials, for example, are age-hardenable alloys; alloy-treated steels; steels containing heterogeneous inclusions; and various types of composite materials in most cases undergoing brittle failure. Most number of modern structural composite materials fail mainly due to the propagation of cracks and this is confirmed by catastrophic consequences of increasing of the cracks in the coverings of welding vessels, planes, in reservoirs, large concrete, and various types of buildings.

Plates with stiffening ribs are widely used in engineering, and, in particular, in manufacturing of aircrafts. By stiffening ribs, we imply a curvilinear elastic rod of constant cross-section (generally speaking, made of elastic material) continuously attached to the plate along its edge, or along any other line, partially or wholly placed inside the physical region. The problem of reinforcing the plates becomes particularly complicated when in the area of reinforcing the base, the medium has such defects as openings, notches, 
cracks, and so forth, and then, the definition of interaction of two opposite factors-its reinforcing and its weakening - takes on special significance.

Problems of contact interaction of thin-walled elements in the form of straight-line stringers with massive deformable bodies of different geometric forms, the results of the investigations which are important for application in the mechanics of composites, in metrical techniques, and in other fields of the applied mechanics were considered by many authors. In Melan's work [16], the problem of the contact of the infinite stringer with an elastic semi-infinite plate, where it is assumed that the rigidity in bending of the stringers is negligibly small, because of which the normal contact stresses are regarded to be equal to zero is first discussed and solved, and finally a model of one-dimensional elastic continuum is admitted for the stringer. Substantiation of this model with exact methods of the two-dimensional theory of elasticity is given in Bufler [6], and in Muki and Sternberg [17] later on. Numerous works forming a rather wide field of the theory of elasticity are dedicated to generalization and development of Melan's results. The basic achievements in this field with sufficient completeness are reflected in the works of Sternberg [19], Erdogan [10], Grigolyuk and Tolkachev [12], Cherepanov [8], Alexandrov and Mkhitaryan [1] and in the book The Development of the Theory of Contact Problems in the USSR [10] edited by Galline. The problems of contact interaction of a discontinuous stringer or thin inclusions with elastic bodies and problems of stress state of massive elastic bodies with cracks, reinforced by stringers, are of great interest from the point of view of the fracture mechanics and are closely connected with the denoted circle of problems. Theoretical investigations of the questions of forming and propagating of cracks (cracking) in the composites are of great interest too. In this direction, we must mention the works of Muki and Sternberg [18], Greif and Sanders Jr. [11], Delale and Erdogan [9], Theocaris et al. [4, 20, 21], Bardzokas et al. [3], and Antipov et al. [2]. In the ideal and methodological aspects, the mentioned problems are highly close to the problems of the mechanics of the contact fracture which are reflected in the works of Lawn and Wilshaw [14], Kolesnikov and Morozov [13], and Cherepanov [7].

Consider the work of Delale and Erdogan [9] more thoroughly in which the question of initiation and propagation of a crack in an elastic half-plane, reinforced on its boundary by the stringer of finite length and uniformly tensioned at infinity, is discussed. On the basis of the analysis of the stress state of the elastic half-plane near the endpoints of the stringer by the criterion of maximum normal discontinuous stresses, the initial possible direction of the crack propagation is determined. It is shown that this direction actually coincides with the perpendicular direction to the half-plane boundary. Later, contact problems on interaction of the elastic half-plane with a finite stringer, containing a vertical inner crack or a vertical crack terminating at the boundary half-plane, are considered. An important particular case of an elastic half-plane, the known length of which is given beforehand, with two symmetrically situated finite stringer and a vertical edge crack, is discussed. In case of a vertical edge crack with the help of the defining system of singular integral equations of the problem and the functional and theoretical method of asymptotic behavior of the integral of Cauchy type near the tips of the integration interval, it is shown that the tangential contact stresses under the stringer and the displacement densities at the endpoint of the stringer and the crack do not have a singularity degree. 


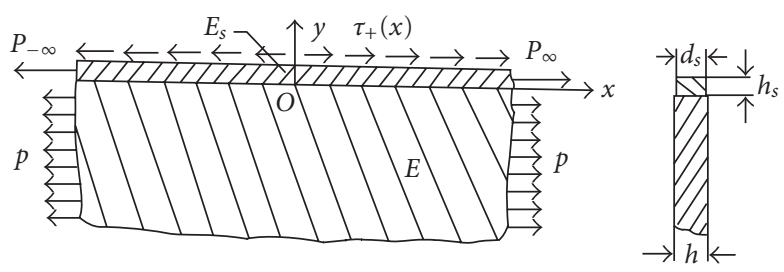

Figure 2.1. Geometry and loading of an elastic semi-infinite plate reinforced on its boundary by the infinite continuous stringer $\left(P_{-\infty}=P_{\infty}\right)$.

In the given work, firstly, we formulate the modified problem of Melan and with the help of Fourier integral transformation, we construct its acoustic solution and then formulate the condition of cracking of an infinite stringer. After that, reveal the necessary restrictions on external loadings, which provide the contact of the failed infinite stringer with a plate without propagation of the crack inside the plate.

\section{Formulation of Melan's modified problem and its solution}

Let an elastic semi-infinite plate related to the right rectangular coordinate $O x y$ (Figure 2.1) with elasticity module $E$ and thickness $h$ on its boundary be reinforced by an infinite stringer with elasticity module $E_{s}\left(E_{s}>E\right)$ and area $A_{s}$ of the cross-section, when $A_{s}=$ $d_{s} h_{s}$, where $d_{s}$ is the width and $h_{s}$ is the height of the stringer. Let then the plate at infinity be tensioned by uniformly distributed forces of intensity $p$ and let the stringer at infinity be stretched by two concentrated forces $P_{ \pm \infty}\left(P_{-\infty}=P_{+\infty}\right)$. Besides, take into account that the tangential forces of intensity $\tau_{+}(x)(-\infty<x<\infty)$ act along the middle line of the top edge of the stringer, when $\tau_{+}(-x)=-\tau_{+}(x)$. It is required to determine the law of distribution of the tangential contact stresses $\tau_{x y}(x, 0)$ and the axial stresses $\sigma_{x}^{s}(x)$ in the stringer.

Assume that the plate is in the generalized plane stress state and the stringer according to the model of one-dimensional elastic continuum is in a uniaxial stress state. Going into the case of the plane deformation is realized by the well-known means.

Introduce the concept of effective width $d=d_{\text {eff }}$ of the contact zone, along which the stringer is actually fastened with the plate. In the particular case when the stringer width is connected with the plate, we have $d=\min \left(d_{s}, h\right)$. When there is a partial connection, $d<d_{s}, h$. In the assumptions made, derive the determining equation of the problem. For this, at first consider the equilibrium of the element, of length $d x$, of the stringer (Figure 2.2).

According to the admitted model of the stringer, the equation of the equilibrium of this element has the form

$$
\frac{d \sigma_{x}^{s}}{d x}=\frac{d \cdot \tau_{-}(x)-\tau_{+}(x)}{A_{s}} \quad(-\infty<x<\infty),
$$

where $\tau_{-}(x)$ denotes the unknown tangential contact stress related to the unit of the effective width $d$ of the contact zone, when $\tau_{-}(-x)=-\tau_{-}(x)$. Then, $\tau_{x y}(x, 0)=d \cdot \tau_{-}(x) / h$. 


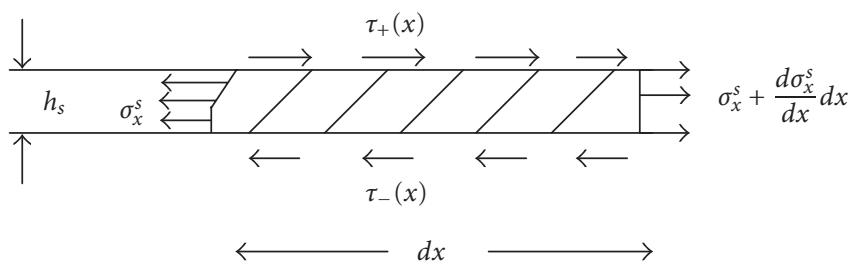

Figure 2.2. Equilibrium of the element of the stringer.

Since

$$
\left.A_{s} \sigma_{x}^{s}(x)\right|_{x= \pm \infty}=P_{ \pm \infty}=P_{\infty}
$$

with the help of the integrity from (2.1), we obtain

$$
\sigma_{x}^{s}(x)=A_{s}^{-1}\left[P_{\infty}-d \int_{x}^{\infty} \tau_{-}(u) d u+\int_{x}^{\infty} \tau_{+}(u) d u\right] \quad(-\infty<x<\infty) .
$$

Here by Hook's law for axial deformations $\varepsilon_{x}^{s}(x)$ of the stringer, we find

$$
\varepsilon_{x}^{s}(x)=E_{s}^{-1} \sigma_{x}^{s}(x)=\left(E_{s} A_{s}\right)^{-1}\left[P_{\infty}-d \int_{x}^{\infty} \tau_{-}(u) d u+\int_{x}^{\infty} \tau_{+}(u) d u\right] \quad(-\infty<x<\infty) .
$$

On the other hand, the horizontal strain component $\varepsilon_{x}(x, 0)$ of boundary points of the elastic semi-infinite plate, when horizontal forces of intensity $\tau_{-}(x)$ are applied on its boundary, normal forces are absent, and uniformly tensile forces $p$ are applied at infinity, is given by the formula derived in the works of Alexandrov and Mkhitaryan [1], and Timoshenko and Goodier [22]:

$$
\varepsilon_{x}(x, 0)=\frac{2 d}{\pi E h} \int_{-\infty}^{\infty} \frac{\tau_{-}(u) d u}{u-x}+\frac{p}{E} \quad(-\infty<x<\infty),
$$

where the integral is understood in the sense of Cauchy principal value.

Now the contact condition $\varepsilon_{x}(x, 0)=\varepsilon_{x}^{s}(x)(-\infty<x<\infty)$, taking into account (2.4) and (2.5), the solution of the produced problem is reduced to the solution of the following integral equation of a convolution type referring to $\tau_{-}(x)$ :

$$
\frac{2 d}{\pi E h} \int_{-\infty}^{\infty} \frac{\tau_{-}(u) d u}{u-x}=\frac{1}{E_{s} A_{s}}\left[-d \int_{x}^{\infty} \tau_{-}(u) d u+\int_{x}^{\infty} \tau_{+}(u) d u\right] \quad(-\infty<x<\infty) .
$$

Here the following relation is taken into account:

$$
\frac{p}{E}=\frac{P_{\infty}}{E_{s} A_{s}}
$$

following from the condition of compatibility of horizontal components of the stringer deformation and plate at infinity, according to (2.4) and (2.5). 
Furthermore in (2.6), we pass to new values, using

$$
\begin{gathered}
x=\frac{E_{s} A_{s}}{E d} \xi, \quad u=\frac{E_{s} A_{s}}{E d} \eta, \quad h_{0}=\frac{h}{d}, \\
\tau_{-}(x)=\tau_{-}\left(\frac{E_{s} A_{s}}{E d} \xi\right)=\tau_{-}^{*}(\xi), \quad \tau_{+}(x)=\tau_{+}\left(\frac{E_{s} A_{s}}{E d} \xi\right)=\tau_{-}^{*}(\xi) .
\end{gathered}
$$

As a result, (2.6) is transformed into the following integro-differential equation of a convolution type of a simpler form:

$$
\frac{2}{\pi} \frac{d}{d \xi} \int_{-\infty}^{\infty} \frac{\tau_{-}^{*}(\eta) d \eta}{\eta-\xi}=h_{0} \tau_{-}^{*}(\xi)-h_{0} d^{-1} \tau_{+}^{*}(\xi) \quad(-\infty<x<\infty) .
$$

Equation (2.9) is solved by a standard method of Fourier integral transform. Introducing Fourier transform

$$
T_{ \pm}^{*}(\lambda)=\int_{-\infty}^{\infty} \tau_{ \pm}^{*}(\xi) \exp (i \lambda \xi) d \xi
$$

from this equation, we easily find the unknown transform

$$
T_{-}^{*}(\lambda)=\frac{h_{0}}{d} \frac{T_{+}^{*}(\lambda)}{h_{0}+2|\lambda|} \quad(-\infty<\lambda<\infty)
$$

Since $\tau_{+}^{*}(\xi)$ and $T_{+}^{*}(\lambda)$ are odd functions, from here by Fourier formula of reverse transform, we obtain

$$
\tau_{-}^{*}(\xi)=\frac{h_{0}}{d} \int_{-\infty}^{\infty} K\left(h_{0}|\xi-\eta|\right) \tau_{+}^{*}(\eta) d \eta \quad(-\infty<\xi<\infty),
$$

where

$$
\begin{aligned}
K(\xi) & =\frac{1}{\pi} \int_{0}^{\infty} \frac{\cos (\lambda \xi) d \lambda}{1+2 \lambda} \\
& =-\frac{2}{2 \pi}\left[\sin \left(\frac{\xi}{2}\right) \operatorname{Si}\left(\frac{\xi}{2}\right)+\cos \left(\frac{\xi}{2}\right) \operatorname{Ci}\left(\frac{\xi}{2}\right)\right] \quad(-\infty<\xi<\infty) .
\end{aligned}
$$

Here $\operatorname{Si}(\xi)$ and $\operatorname{Ci}(\xi)$ are well-known integral sine and cosine functions, see Bateman and Erdélyi [5],

$$
\operatorname{Si}(\xi)=-\int_{\xi}^{\infty} \frac{\sin u}{u} d u, \quad \operatorname{Ci}(\xi)=-\int_{\xi}^{\infty} \frac{\cos u}{u} d u .
$$

Now in formula (2.12), return to the previous values by (2.8). As a result, we have

$$
\tau_{-}(x)=\frac{h_{0}}{c d} \int_{-\infty}^{\infty} K\left(\frac{h_{0}}{c}|x-u|\right) \tau_{+}(u) d u \quad(-\infty<x<\infty)\left(c=\frac{E_{s} A_{s}}{E d}\right) .
$$

Further consider a particular case of the external loading $\tau_{+}(x)$ when at points $x= \pm a$ of the top edge of the stringer, there are two opposite directed horizontal concentrated 
forces $P$, that is when

$$
\tau_{+}(x)=P d[\delta(x-a)-\delta(x+a)] \quad(0<a<\infty),
$$

where $\delta(x)$ is Dirac's well-known Delta function. Admit also that $d_{s}=d=h$. Then $h_{0}=1$ and formula (2.15) will have the following form:

$$
\tau_{-}(x)=\frac{P}{c}\left[K\left(\frac{|x-a|}{c}\right)-K\left(\frac{|x+a|}{c}\right)\right] \quad(-\infty<x<\infty) .
$$

Now using (2.16) and (2.17) in formula (2.3) and taking into account relation (2.7) for the axial stresses in the stringer, we get

$$
\begin{aligned}
\sigma_{x}^{s}(x)= & x p-\frac{P}{\chi h_{s}^{2}} \int_{x}^{\infty}\left[K\left(\frac{|u-a|}{c}\right)-K\left(\frac{|u+a|}{c}\right)\right] d u \\
& -\frac{P}{h_{s}}[H(x-a)-H(x+a)], \quad-\infty<x<\infty ; c=\chi h_{s}, \chi=\frac{E_{s}}{E},
\end{aligned}
$$

where $H(x)$ is Heaviside's function. Since the maximum tensile stresses are reached in the stringer in its section, $x=0$ according to (2.18), we find

$$
\max \sigma_{x}^{s}=\sigma_{x}^{s}(0)=\chi p-\frac{P}{\chi h_{s}^{2}} \int_{x}^{\infty}\left[K\left(\frac{|u-a|}{c}\right)-K\left(\frac{|u+a|}{c}\right)\right] d u+\frac{P}{h_{s}} .
$$

The integral entering this formula with the help of the integral presentation of function $K(\xi)$ from (2.13) will obtain the following form:

$$
\begin{aligned}
I(a) & =\int_{0}^{\infty}\left[K\left(\frac{|u-a|}{c}\right)-K\left(\frac{|u+a|}{c}\right)\right] d u \\
& =\frac{1}{\pi} \int_{0}^{\infty} d u \int_{0}^{\infty} \frac{\cos [\lambda(\eta-\alpha)]}{1+2 \lambda} d \lambda-\frac{1}{\pi} \int_{0}^{\infty} d u \int_{0}^{\infty} \frac{\cos [\lambda(\eta+\alpha)]}{1+2 \lambda} d \lambda \\
& =\frac{2 c}{\pi} \lim _{N \rightarrow \infty} \int_{0}^{N} d \eta \int_{0}^{\infty} \frac{\sin (\lambda \eta) \sin (\lambda \xi)}{1+2 \lambda} d \lambda \quad\left(\eta=\frac{u}{c}, \alpha=\frac{a}{c}\right) .
\end{aligned}
$$

Changing the order of the integration and using the Rieman-Lebege theorem (see Titchmarsh [23]), we have

$$
\begin{gathered}
I(a)=c[1+2 \omega(\alpha)] \quad\left(\alpha=\frac{a}{c}=\frac{a}{\chi h_{s}}\right) \\
\omega(\alpha)=-\frac{2}{\pi} \int_{0}^{\infty} \frac{\sin (\lambda \alpha)}{1+2 \lambda} d \lambda=\frac{1}{\pi}\left[\cos \left(\frac{\alpha}{2}\right) \operatorname{Si}\left(\frac{\alpha}{2}\right)-\sin \left(\frac{\alpha}{2}\right) \operatorname{Ci}\left(\frac{\alpha}{2}\right)\right] .
\end{gathered}
$$

Therefore, formula (2.19) with the help of (2.21) is transformed into the form

$$
\max \sigma_{x}^{s}=\sigma_{x}^{s}(0)=\chi p-2 P h_{s}^{-1} \omega(\alpha) .
$$

On the base of this equality, we can write the cracking condition of the stringer

$$
\chi p-2 P h_{s}^{-1} \omega(\alpha)=\sigma_{s}
$$


where $\sigma_{s}$ is the brittle strength limit of the material of the stringer under the tension, and the function $\omega(\alpha)$ is given in (2.21). This function by the well-known theorem of Fourier integral theory (see Titchmarsh [23]) is not positive, but the right-hand side of (2.22) or the left-hand side of (2.23) is always positive.

The condition (2.23) can be written in the nondimensional form

$$
P_{0}=-\frac{k-\chi}{2 \omega(\alpha)} \quad\left(P_{0}=\frac{P}{h_{s} p}, k=\frac{\sigma_{s}}{p}\right) .
$$

Thus, when the external loadings are subjected to condition (2.23) or (2.24), a brittle rupture of the stringer takes place.

Note that if $\tau_{+}(x) \equiv 0$, the homogeneous equation (2.6) or (2.9) has only the trivial zero solution $\tau_{-}(x)=\tau_{-}^{*}(\xi) \equiv 0$ and on the bases of (2.3) and (2.7), we get

$$
\sigma_{x}^{s}(x)=\frac{P_{\infty}}{A_{s}}=p \chi
$$

Then the condition (2.23) goes over to the condition $\chi p=\sigma_{s}$.

\section{Contact of a ruptured infinite stringer with an elastic semi-infinite plate}

Consider the following stage of development of the above-discribed contact problem, when the acting external forces satisfy condition (2.23) or the analogous condition in case of general loading $\tau_{+}(x)$. In this way, we come to the problem of a contact interaction of a ruptured infinite stringer with an elastic semi-infinite plate (Figure 3.1). In the same statement, which was above, we can derive the determining equation of the problem.

Since in the discussed case $\sigma_{x}^{s}(0)=0$, from $(2.1)$, we get

$$
\sigma_{x}^{s}(x)=A_{s}^{-1}\left[d \int_{0}^{x} \tau_{-}(u) d u-\int_{0}^{x} \tau_{+}(u) d u\right] \quad(-\infty<x<\infty) .
$$

The equality (3.1) can also be obtained immediately considering the equilibrium of portion $[0, x]$ of the right part of the stringer and taking into account the oddness of function $\tau_{ \pm}(x)$. It is evident that $\sigma_{x}^{s}(x)$ is the even function. On the other hand, since the stringer at infinity is tensioned by concentrated forces $P_{ \pm \infty}=P_{\infty}$, fulfilling in (3.1) the limiting transition $x \rightarrow \infty$, we will come to the following equality:

$$
d \int_{0}^{\infty} \tau_{-}(u) d u-\int_{0}^{\infty} \tau_{+}(u) d u=P_{\infty}
$$

and here relation (2.7) again takes place.

Thus, in the discussed problem, unknown tangential contact stresses $\tau_{-}(x)$ must be subjected to condition (3.2).

Further by Hook's law instead of (2.4), we will have

$$
\varepsilon_{x}^{s}(x)=E_{s}^{-1} \sigma_{x}^{s}(x)=\left(E_{s} A_{s}\right)^{-1}\left[d \int_{0}^{x} \tau_{-}(u) d u-\int_{0}^{x} \tau_{+}(u) d u\right] \quad(-\infty<x<\infty),
$$




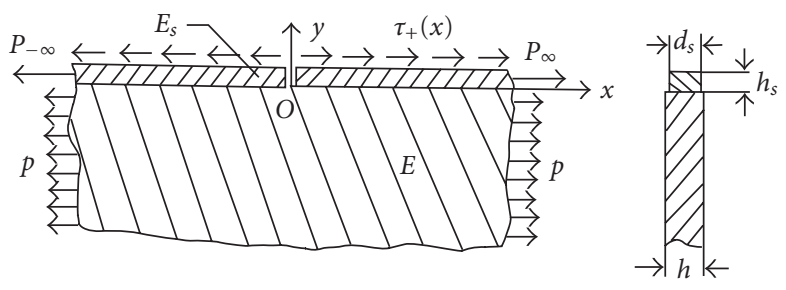

Figure 3.1. Geometry and loading of an elastic semi-infinite plate reinforced on its boundary by the infinite discontinuous stringer $\left(P_{-\infty}=P_{\infty}\right)$.

and $\varepsilon_{x}(x, 0)$ of the plate is again expressed by the formula (2.5). The condition of contact $\varepsilon_{x}(x, 0)=\varepsilon_{x}^{s}(x)(-\infty<x<\infty)$ reduces to the following integral equation in the given case

$$
\frac{2 d}{\pi E h} \int_{-\infty}^{\infty} \frac{\tau_{-}(u) d u}{u-x}+\frac{p}{E}=\frac{1}{E_{s} A_{s}}\left[d \int_{0}^{x} \tau_{-}(u) d u-\int_{0}^{x} \tau_{+}(u) d u\right] \quad(-\infty<x<\infty),
$$

the solution of which must satisfy condition (3.2). Using this condition and (2.7), it is easy to show that (2.6) and (3.4) are identical and, therefore, have the same solution (2.15).

However, taking into account the peculiarity of the given problem determining the integral equation, we will formulate the determinative integral equation on semiaxis $(0, \infty)$ and apply to it the means of Fourier integral sine transform. Such a way of solving of the equation is necessary for substantiating some facts for their use in the future.

For this purpose at first in (3.4), we will go over to new variables by formula (2.8). Then this equation will be transformed to the form

$$
\frac{2}{\pi} \int_{-\infty}^{\infty} \frac{\tau_{-}^{*}(\eta) d \eta}{\eta-\xi}=h_{0} \int_{0}^{\xi} \tau_{-}^{*}(\eta) d \eta-h_{0} d^{-1} \int_{0}^{\xi} \tau_{+}^{*}(\eta) d \eta-h_{0} p \quad(-\infty<\xi<\infty),
$$

and condition (3.2) allowing for (2.7) will be transformed to the form

$$
\int_{0}^{\infty} \tau_{-}^{*}(\eta) d \eta-d^{-1} \int_{0}^{\infty} \tau_{+}^{*}(\eta) d \eta=p
$$

Then putting

$$
\begin{aligned}
X(\xi) & =h_{0} \int_{0}^{\xi} \tau_{-}^{*}(\eta) d \eta-h_{0} d^{-1} \int_{0}^{\xi} \tau_{+}^{*}(\eta) d \eta-h_{0} p \\
& =-h_{0} \int_{\xi}^{\infty} \tau_{-}^{*}(\eta) d \eta+h_{0} d^{-1} \int_{\xi}^{\infty} \tau_{+}^{*}(\eta) d \eta \quad(-\infty<\xi<\infty),
\end{aligned}
$$

we write (3.5) in the form

$$
\frac{2}{\pi} \int_{-\infty}^{\infty} \frac{X^{\prime}(\eta) d \eta}{\eta-\xi}=h_{0} X(\xi)-\frac{2 h_{0}}{\pi d} \int_{-\infty}^{\infty} \frac{\tau_{+}^{*}(\eta) d \eta}{\eta-\xi} \quad(-\infty<\xi<\infty) .
$$

Hence by the formula of Hilbert reverse transform, we find

$$
X^{\prime}(\xi)=-\frac{h_{0}}{2 \pi} \int_{-\infty}^{\infty} \frac{X(\eta) d \eta}{\eta-\xi}-\frac{h_{0}}{d} \tau_{+}^{*}(\xi) \quad(-\infty<\xi<\infty) .
$$


If now we make integration by parts in the last integral with Cauchy kernel and take into account the property of oddness of functions $\tau_{ \pm}^{*}(\xi)$, we will arrive at the following integral equation on the positive semiaxis with respect to function $\tau_{-}^{*}(\xi)$ :

$$
\tau_{-}^{*}(\xi)+\frac{h_{0}}{2 \pi} \int_{0}^{\infty} \ln \frac{\xi+\eta}{|\xi-\eta|} \tau_{-}^{*}(\eta) d \eta=\frac{h_{0}}{2 \pi d} \int_{0}^{\infty} \ln \frac{\xi+\eta}{|\xi-\eta|} \tau_{+}^{*}(\eta) d \eta \quad(0<\xi<\infty) .
$$

Here integrating by parts, it is taken into account that according to (3.7), at least $X(\xi)=$ $O\left(|\xi|^{-\varepsilon}\right)(\xi \rightarrow \infty, \varepsilon>0)$.

For solving integral equation (3.10), we will use the spectral relation

$$
\frac{1}{\pi} \int_{0}^{\infty} \ln \frac{\xi+\eta}{|\xi-\eta|} \sin \lambda \eta d \eta=\frac{\sin \lambda \xi}{\lambda} \quad(\xi>0, \lambda>0),
$$

following from the well-known formula given in the book by Bateman and Erdélyi [5, page 77 , formula 2.6(1)] and, besides, for the further calculations, we introduce

$$
\tau_{ \pm}^{*}(\xi)=\int_{0}^{\infty} T_{ \pm}(\lambda) \sin \lambda \xi d \lambda
$$

where $T_{+}(\lambda)$ is the known function and $T_{-}(\lambda)$ is the unknown function. Later we introduce the expressions $\tau_{ \pm}^{*}(\xi)$ from (3.12) into (3.10) and change the order of the integration taking into account relation (3.11). In the result, we find

$$
T_{-}(\lambda)=\frac{h_{0}}{d} \frac{T_{+}(\lambda)}{h_{0}+2 \lambda} \quad(0<\lambda<\infty)
$$

and therefore, by (3.12), we will have

$$
\tau_{-}^{*}(\xi)=\frac{h_{0}}{d} \int_{0}^{\infty}\left\{K\left[h_{0}|\xi-\eta|\right]-K\left[h_{0}(\xi+\eta)\right]\right\} \tau_{+}^{*}(\eta) d \eta \quad(0<\xi<\infty),
$$

where function $K(\xi)$ is given by formula (2.13). The obtained solution (3.14) of the integral equation (3.10) coincides with (2.12) obtained above.

Taking into account solution (3.14), condition (3.6) transforms into

$$
d^{-1} \int_{0}^{\infty}\left\{h_{0} \int_{0}^{\infty}\left[K\left(h_{0}|\xi-\eta|\right)-K\left(h_{0}(\xi+\eta)\right)\right] d \xi-1\right\} \tau_{+}^{*}(\eta) d \eta=p .
$$

Thus the solution of problem (3.14) must be subjected to condition (3.15).

Now we must note that according to (3.11), the spectrum introduced into (3.10) integral operator considered in space $L_{1}(0, \infty)$ continuously fills positive semiaxis $(0, \infty)$ and that is why, when $\tau_{+}(x)=\tau_{+}^{*}(\xi) \equiv 0$, this homogeneous integral equation has a unique zero solution, which, of course, does not satisfy condition (3.6) or (3.15). Therefore, the problem of the contact of a discontinuous infinite stringer with an elastic semi-infinite plate, when only uniformly tensioned at infinity forces affect the system of stringer-plate, does not have a solution unlike the case of continuous stringer.

Further, the question of existence of the solution will be discussed in case of particular load (2.16) and for the simplicity, we will have $h_{0}=1$, as above. Then after returning 
to the previous variables by (2.8), formula (3.14) will give solution (2.17), and solution (3.15) will turn into the following:

$$
P \int_{0}^{\infty}\left[K\left(\frac{|x-a|}{c}\right)-K\left(\frac{x+a}{c}\right)\right] d x=c(P+c p) \quad\left(c=\chi h_{s}=\frac{h_{s} E_{s}}{E}\right) .
$$

Later transforming this relation with the help of (2.21a) and (2.21b), we will get

$$
2 P_{0} \omega(\alpha)=\chi \quad\left(\chi=\frac{E_{s}}{E}, P_{0}=\frac{P}{p h_{s}}\right) .
$$

Thus, in case of the discussed particular load, the solution of problem (2.17) must satisfy condition (3.17). But it is evident that following the dispositiveness of the function $\omega(\alpha)$, this solution cannot be satisfied and, therefore, the problems again do not have solutions.

But, nevertheless, in order to get the fulfillment of condition (3.17) to available load (2.16), we will add another similar pair of horizontal concentrated forces $P_{1}$ placed at points $x= \pm a_{1}$ on the top edge of the stringer with opposite direction to the one of the initial pair of forces $P$, therefore instead of (2.16), we will consider the following load:

$$
\tau_{+}(x)=P d[\delta(x-a)-\delta(x+a)]-P_{1} d\left[\delta\left(x-a_{1}\right)-\delta\left(x+a_{1}\right)\right] .
$$

Then instead of problem (2.17), we will have the following solution $(-\infty<x<\infty)$ :

$$
\tau_{-}(x)=\frac{P}{c}\left[K\left(\frac{|x-a|}{c}\right)-K\left(\frac{x+a}{c}\right)\right]-\frac{P_{1}}{c}\left[K\left(\frac{\left|x-a_{1}\right|}{c}\right)-K\left(\frac{x+a_{1}}{c}\right)\right],
$$

and instead of solution (3.17), we will have the following condition

$$
2\left[P_{0} \omega(\alpha)-P_{1}^{*} \omega\left(\alpha_{1}\right)\right]=\chi \quad\left(\chi=\frac{E_{s}}{E}\right)
$$

where

$$
\alpha=\frac{a}{c}=\frac{a}{\chi h_{s}}, \quad \alpha_{1}=\frac{a_{1}}{c}=\frac{a_{1}}{\chi h_{s}}, \quad P_{0}=\frac{P}{p h_{s}}, \quad P_{1}^{*}=\frac{P_{1}}{p h_{s}},
$$

and function $\omega(\alpha)$ is given by formula (2.21b). It is evident, that by a suitable choice of the parameters $P_{0}, P_{1}^{*}, \alpha$, and $\alpha_{1}$, it is possible to obtain the satisfaction of condition (2.4). Note that $\omega(\alpha) \leq 0$ when $(0<\alpha<\infty)$ and $\omega(\alpha) \rightarrow 0$ when $\alpha \rightarrow 0$.

Performing (3.20), we reveal the asymptotic behavior of contact stresses $\tau_{-}(x)$ from (3.19) in the vicinity of point $x=0$. With this purpose, we will observe that with the help of (2.13) we can represent formula (3.19), in the form of

$$
\begin{gathered}
\tau_{-}(x)=\frac{2}{\pi c}\left[P \int_{0}^{\infty} \frac{\sin (\lambda x / c) \sin (\lambda \alpha)}{1+2 \lambda} d \lambda-P_{1} \int_{0}^{\infty} \frac{\sin (\lambda x / c) \sin \left(\lambda \alpha_{1}\right)}{1+2 \lambda} d \lambda\right] \\
\left(0<x<\infty ; c=\chi h_{s}=\frac{h_{s} E_{s}}{E}\right) .
\end{gathered}
$$


Since

$$
\begin{aligned}
\frac{\sin (\lambda \alpha)}{1+2 \lambda} & =\sin (\lambda \alpha)(1+2 \lambda)^{-1}=\frac{\sin (\lambda \alpha)}{2 \lambda}\left(1-\frac{1}{2 \lambda}+\frac{1}{4 \lambda^{2}}-\cdots\right) \\
& \approx \frac{\sin (\lambda \alpha)}{2 \lambda}(\lambda \longrightarrow \infty),
\end{aligned}
$$

by the well-known results reflected in the works of Lighthill [15] and according to (3.11), we will get

$$
\begin{aligned}
\tau_{-}(x) & \approx \frac{P}{2 \pi c} \ln \frac{\alpha+x / c}{|\alpha-x / c|}-\frac{P_{1}}{2 \pi c} \ln \frac{\alpha_{1}+x / c}{\left|\alpha_{1}-x / c\right|} \\
& =\frac{P}{2 \pi c} \ln \frac{1+x / a}{|1-x / a|}-\frac{P_{1}}{2 \pi c} \ln \frac{1+a_{1}}{\left|1-x / a_{1}\right|} \quad(x \longrightarrow 0) .
\end{aligned}
$$

Then taking into consideration the known MacLaurin series for function $\ln (1+x)(|x|<1)$, we finally find

$$
\tau_{-}(x) \approx\left(\frac{P}{a}-\frac{P_{1}}{a_{1}}\right) \frac{x}{\pi c} \quad(x \longrightarrow 0)
$$

from which it follows that when observing condition (3.20), the contact stresses at the point of the stringer break are equal to zero.

On the basis of this statement, it may be confirmed that the solution of the problem of the contact of an infinite discontinuous stringer with an elastic semi-infinite plate, generally, does not exist. It exists only at observance of condition (3.15) or in particular, of condition (3.20) and then the problem has the solution in form of (3.14) or (2.15) or (3.19), correspondingly, which coincides with the corresponding solution in case of the infinite continuous stringer. It means that at restriction of type (3.15) or (3.20) on the external forces, the contact of the discontinuous infinite stringer with the plate is possible without propagation of the crack into the inner part of the plate. In the opposite case, when these restrictions are not maintained after the stringer cracking, the crack is sure to be propagated into the inner part of the plate. In that case, a new problem arises, which may be of interest for further investigations.

It must be noticed that the indicated difficulties, connected with the question of the existence of the problem solution, are removed if we define the model of one-dimensional elastic continuum of the stringer and consider the discontinuous stringer within the limits of two-dimensional theory of elasticity as two half-planes.

\section{Conclusions}

In case of particular load (2.16) for calculation of nondimensional force $P_{0}$ by formula (2.24), it is necessary to admit that $k=10$ for a brittle cracking of the continuous stringer. The results of the calculations by this formula for different values of parameters $\chi$ and $h_{s} / a$ are given in Table 4.1. Parameter $\chi$ characterizes the relative rigidity of the stringerplate system and parameter $h_{s} / a$ is the relative distance of right-concentrated force $P$ from the origin of coordinates. The analysis of the data of Table 4.1 shows that by fixed 
Table 4.1. Variation of the undimensional force $P_{0}=-((k-\chi) / 2 \omega(\alpha))\left(P_{0}=P / h_{s} p, k=\sigma_{s} / p, \alpha=\right.$ $\left.a / \chi h_{s}\right)$ necessary for the brittle cracking of the continuous infinite stringer depending on related rigidity of the stringer-plate system $\chi=E_{s} / E$ and on the geometric parameter $h_{s} / a$ when at the points $x= \pm a$ of the top edge of the stringer, there act two equal and opposite directed horizontal concentrated forces $P$ (formula (2.24)).

\begin{tabular}{c|cccccccc}
\hline \multirow{2}{*}{$h_{s} / a$} & \multicolumn{7}{|c}{$\chi$} & \\
\cline { 2 - 9 } & 1 & 2 & 3 & 4 & 5 & 6 & 7 & 8 \\
\hline 0.03 & 474.573 & 214.850 & 128.615 & 85.218 & 58.684 & 40.452 & 26.895 & 16.222 \\
0.05 & 287.993 & 133.582 & 82.157 & 55.850 & 39.366 & 27.707 & 18.766 & 11.509 \\
0.07 & 208.822 & 99.504 & 62.755 & 43.583 & 31.277 & 22.351 & 15.337 & 9.512 \\
0.1 & 150.280 & 74.467 & 48.487 & 34.526 & 25.276 & 18.358 & 12.769 & 8.010 \\
0.3 & 62.340 & 36.717 & 26.662 & 20.469 & 15.840 & 12.010 & 8.645 & 5.579 \\
\hline
\end{tabular}

quantity $\chi$ with decreasing of parameter $h_{s} / a$, that is, with increasing of distance $a$, value $P_{0}$ increases. It means that with the removal of concentrated tensioned forces $P$ from the origin of coordinates for the stringer breaking, more forces will be required. On the contrary, by fixed value $h_{s} / a$ with the increase of the parameter $\chi$, a decrease of the value $P_{0}$ takes place.

In conclusion, we should underline that in the problem considered here, we simulate the problem of cracking of a two-layered composite of a rectangular form. The problem of such type with mixed boundary conditions theoretically brings us to new statements and extends the class of the boundary problems of the mathematical theory of elasticity and of adjacent branches of the mechanics of solid media. As to the applied sciences, such problems may be encountered in various fields of the applied mechanics, for example, in the mechanics of composites, in structural mechanics, and so forth. The investigations of the analogous problems are of particular interest theoretically as well as practically, and can be carried out by the methods similar to those that are used in the given work.

\section{References}

[1] V. M. Alexandrov and S. M. Mkhitaryan, Contact Problems for Bodies with Thin Coverings and Layers, Nauka, Moscow, 1983 (Russian).

[2] Y. A. Antipov, D. Bardzokas, and G. E. Exadaktylos, Partially stiffened elastic half-plane with an edge crack, Internat. J. Fracture 85 (1997), 241-263.

[3] D. Bardzokas, G. E. Exadaktylos, and G. Anastaselos, The effect of stringers and patches on the stress intensities around cracks in plates, Engrg. Fracture Mech. 55 (1996), no. 6, 935-955.

[4] D. Bardzokas, V. Z. Parton, and P. S. Theocaris, Integral equations in elasticity theory for a multiply connected domain with inclusions, Prikl. Mat. Mekh. 53 (1989), no. 3, 485-495 (Russian).

[5] H. Bateman and A. Erdélyi, Higher Transcendental Functions. Vol. II, McGraw-Hill Book Company, New York, 1953.

[6] H. Bufler, Zur Krafteinleitung in Scheiben über geschweisste oder geklebte Verbindungen, Österreich Ing.-Arch. 18 (1964), no. 3-4, 284-292 (German).

[7] G. P. Cherepanov, A problem of stamp indentation with formation of cracks, J. Appl. Math. Mech. 27 (1963), no. 1, 210-216.

[8]__ Fracture Mechanics of Composite Materials, Nauka, Moscow, 1983 (Russian).

[9] F. Delale and F. Erdogan, The crack problem for a half plane stiffened by elastic cover plates, Internat. J. Solids Structures 18 (1982), no. 5, 381-395. 
[10] F. Erdogan, Analysis of elastic over plates. Developments in mechanics, Proceedings of the 12th Meidwestern Mechanics Conference (1971), The development of theory of contact problems in USSR, vol. 6, Nauka, Moscow, 1976, pp. 817-830 (Russian).

[11] R. Greif and J. L. Sanders, Jr., The effect of a stringer on the stress in a cracked sheet, Trans. ASME J. Appl. Mech. 32 (1965), no. 1, 59-66, Russian translation.

[12] E. I. Grigolyuk and V. M. Tolkachev, Contact Problems of Theory of Plates and Shells, Mashinostroenie, Moscow, 1980 (Russian).

[13] U. V. Kolesnikov and E. M. Morozov, Mechanics of Contact Fracture, Nauka, Moscow, 1989 (Russian).

[14] B. R. Lawn and T. R. Wilshaw, Indentation fracture: principles and application, J. Mater. Sci. 10 (1975), no. 6, 1049-1081.

[15] M. J. Lighthill, Introduction to Fourier Analysis and Generalized Functions, Cambridge University Press, New York, 1958.

[16] E. Melan, Ein Beitrag zur Theorie geschweisster Verbindungen, Ing.-Arch. 3 (1932), no. 2, 123129 (German).

[17] R. Muki and E. Sternberg, Transfer of load from an edge-stiffener to a sheet-a reconsideration of Melan's problem, Trans. ASME J. Appl. Mech. 34 (1967), no. 3, 679.

[18] Load-absorption by a discontinuous filament in a fiber-reinforced composite, Z. Angew. Math. Phys. 22 (1971), 809-824.

[19] E. Sternberg, Load-transfer and load-diffusion in elastostatics, Proceedings of the Sixth U.S. National Congress of Applied Mechanics, 1970, p. 34.

[20] P. S. Theocaris and D. Bardzokas, The influence of a finite stringer on the stress intensities around cracks in plates, Engrg. Fracture Mech. 14 (1981), 493-508.

[21] The frictionless contact of cracked elastic bodies, ZAMM Z. Angew. Math. Mech. 63 (1983), no. 2, 89-102.

[22] S. P. Timoshenko and J. N. Goodier, Theory of Elasticity, McGraw-Hill Book Company, New York, 1970.

[23] E. C. Titchmarsh, An Introduction of the Theory of Fouriery Integrals, 2nd ed., University of Oxford, 1948.

D. I. Bardzokas: Laboratory of Strength and Materials, Department of Mechanics, Faculty of Applied Mathematics and Physics Sciences, National Technical University of Athens, Zographou Campus, Theocaris Buildings, Athens 15773, Greece

E-mail address: bardim@central.ntua.gr

G. I. Sfyris: Laboratory of Strength and Materials, Department of Mechanics, Faculty of Applied Mathematics and Physics Sciences, National Technical University of Athens, Zographou Campus, Theocaris Buildings, Athens 15773, Greece

E-mail address: gsfyris@mail.ntua.gr 


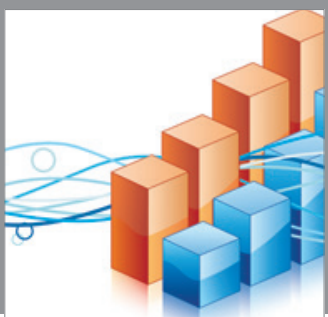

Advances in

Operations Research

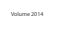

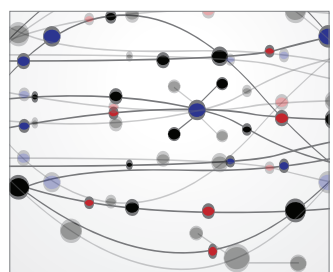

\section{The Scientific} World Journal
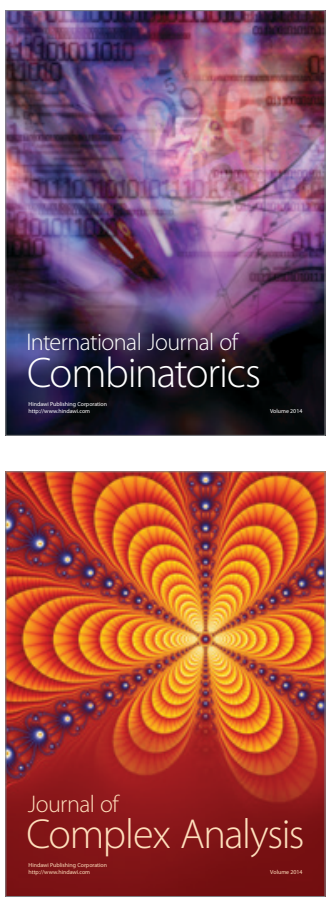

International Journal of

Mathematics and

Mathematical

Sciences
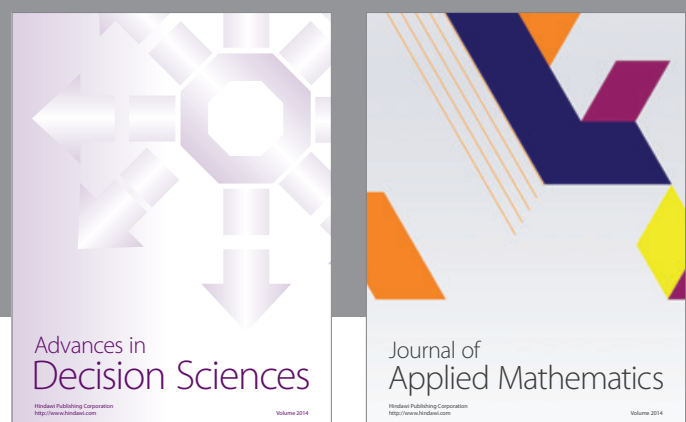

Journal of

Applied Mathematics
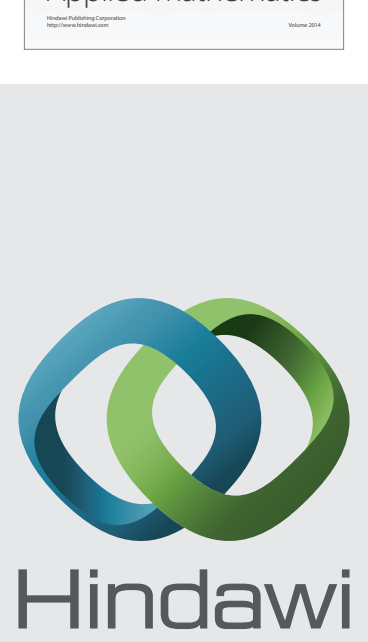

Submit your manuscripts at http://www.hindawi.com
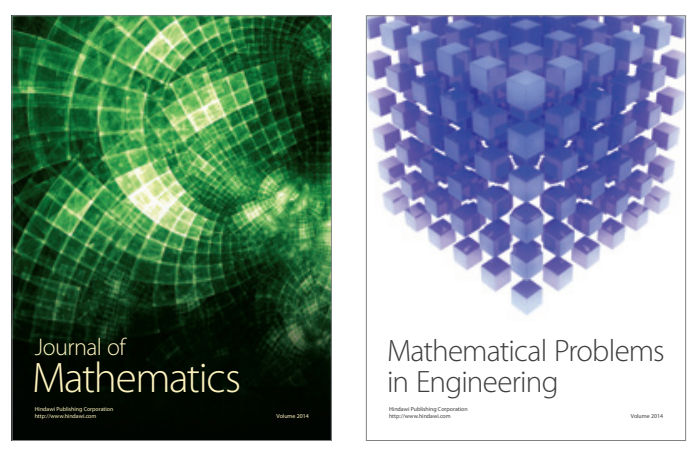

Mathematical Problems in Engineering
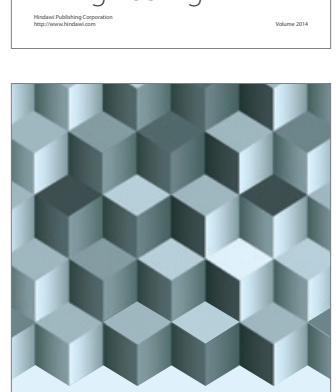

Journal of

Function Spaces
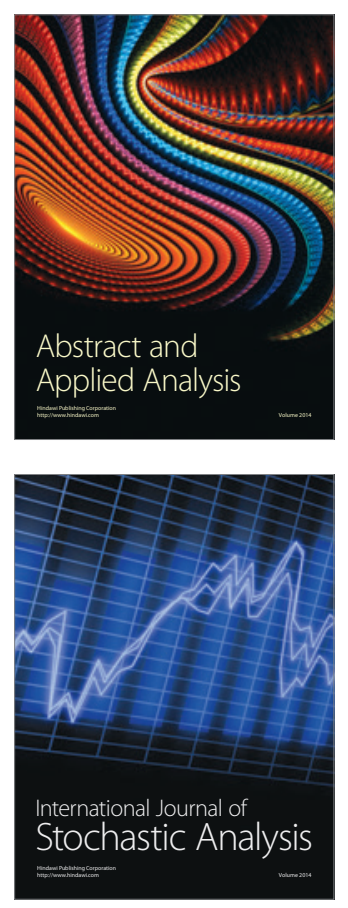

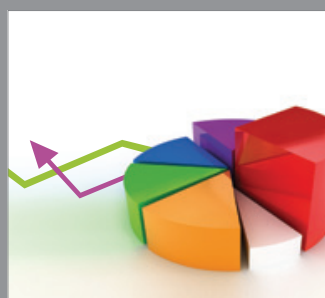

ournal of

Probability and Statistics

Promensencen
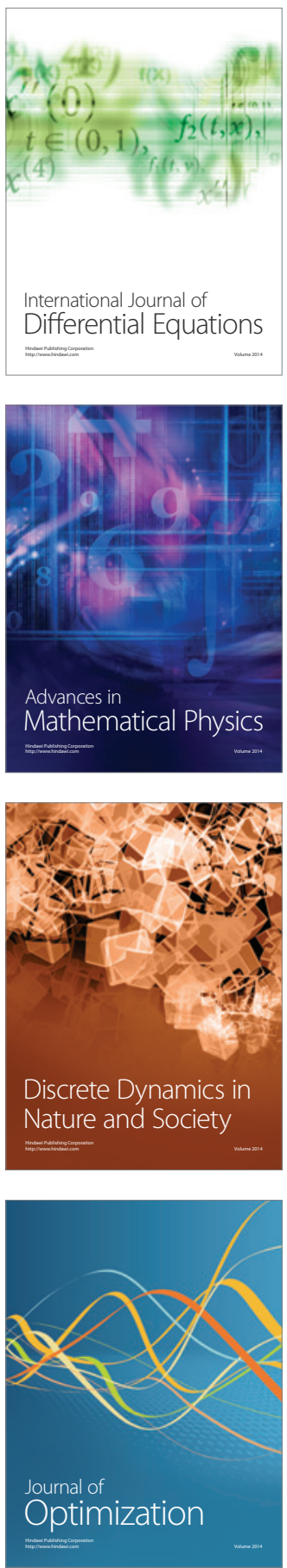